\title{
Paths toward implementing eDNA-based bioassessments across the United States
}

\author{
Joseph Craine ${ }^{\ddagger}$, Nicholas Schulte $\ddagger$ \\ $\ddagger$ Jonah Ventures, Boulder, United States of America
}

Corresponding author: Nicholas Schulte (nicholas.o.schulte@gmail.com)

Received: 24 Feb 2021 | Published: 04 Mar 2021

Citation: Craine J, Schulte N (2021) Paths toward implementing eDNA-based bioassessments across the United States. ARPHA Conference Abstracts 4: e64995. https://doi.org/10.3897/aca.4.e64995

\begin{abstract}
In the United States, bioassessment is a key component of clean water assessment for each state and territory under federal guidelines. Yet, there is a patchwork of approaches implemented by different states that restricts geographic comparisons of water condition. At Jonah Ventures, we have reviewed approaches to implementation of bioassessment for each state and have recommendations for the adoption of aquatic eDNA metabarcoding to complement or replace current practices. States sample different organisms and assess biotic condition differently. For example, only 38 of 55 U.S. states and territories use or have developed methods for using fish assemblages in bioassessments of wadeable streams and rivers. States also differ in the analytical methods used to determine impairment. For benthic macroinvertebrate-based assessments, 2 states apply biotic indices, 35 multimetric indices, 2 predictive models, 2 no standard, and 9 a combination of approaches. Additionally, states vary in ecological values assigned to species, a result of geographic and methodological discrepancies. For instance, the common carp is considered tolerant of disturbance in most states, but not in Arkansas, Georgia, or Maryland.
\end{abstract}

Adoption of eDNA-based bioassessments has the benefits of being cheaper and faster while providing higher taxon detection and resolution than traditional techniques. A single sample can be used to detect the presence or relative abundances of multiple biotic groups. As a result, sample collection can be democratized, and larger, more frequent datasets can be generated that cover a greater range of environments. 
As a first effort, in 2020 , we collected over 100 filtered water samples from streams across the conterminous states. We sequenced multiple PCR replicates of each sample for a hypervariable region of the 12S rRNA gene using the MiFish-U primer set targeting bony fish. ESVs were then assigned to morphospecies taxonomy, and we calculated morphospecies trait-based and compositional metrics for each sample. Up to 18 species of fish were identified at a single site. We also began the process of developing de novo sequence-based ecological values and indices.

Given recent advances in sample collection and processing, the path to adoption of eDNAbased assessment in the U.S. is clear:

(1) Primer sets for benthic macroinvertebrates need to be improved.

(2) Reference sequence libraries need to be expanded. As an example, only about onethird of the freshwater fish species in the U.S. have been sequenced to date. Although taxonomy-free assessments are viable, empirically managers will require taxon-specific information prior to adoption. Genome skimming is recommended for constructing full mitochondrial and chloroplast genome sequence libraries.

(3) eDNA must be sampled across a broad gradient of sites that vary in their biogeography and stressors. We estimate about 10,000 samples across the U.S. should be sufficient to generate high-performing indices across the various ecoregions.

(4) Analytical techniques must be rigorously tested, regionally calibrated, and consistently implemented across states. We favor an approach in which indices are calculated from gradients of ESV or taxon presence or relative abundances that can secondarily be interpreted based on specific ecological conditions - as opposed to a generalized stress gradient.

The transition to eDNA-based bioassessments in the U.S. can likely be accomplished for

\section{Keywords}

algae, benthic macroinvertebrates, biotic index, eDNA, fish, genome skimming, metabarcoding, United States

\section{Presenting author}

Joseph Craine

\section{Presented at}

1st DNAQUA International Conference (March 9-11, 2021) 


\section{Hosting institution}

Jonah Ventures 